\section{Activin type IIA and IIB receptors mediate Gdf11 signaling in axial vertebral patterning}

\author{
S. Paul Oh, ${ }^{1,6}$ Chang-Yeol Yeo, ${ }^{2,5}$ Youngjae Lee, ${ }^{1}$ \\ Heindrich Schrewe, ${ }^{3}$ Malcolm Whitman, ${ }^{2}$ \\ and $\mathrm{En}^{\mathrm{Li}^{4}}$
}

\begin{abstract}
${ }^{1}$ Department of Physiology and Functional Genomics, University of Florida College of Medicine, Gainesville, Florida 32610, USA; ${ }^{2}$ Department of Cell Biology, Harvard Medical School, Boston, Massachusetts 02115, USA; ${ }^{3}$ Department of Developmental Biology, Max-Planck-Institut für Immunbiologie, Freiburg, Germany; ${ }^{4}$ Cutaneous Biology Research Center and Cardiovascular Research Center, Harvard Medical School, Massachusetts General Hospital-East, Charlestown, Massachusetts 02129, USA
\end{abstract}

Vertebral bodies are segmented along the anteroposterior (AP) body axis, and the segmental identity of the vertebrae is determined by the unique expression pattern of multiple Hox genes. Recent studies have demonstrated that a transforming growth factor $\beta$ (TGF- $\beta$ ) family protein, Gdf11 (growth and differentiation factor 11), and the activin type II receptor, ActRIIB, are involved in controlling the spatiotemporal expression of multiple Hox genes along the AP axis, and that the disruption of each of these genes causes anterior transformation of the vertebrae. Skeletal defects are more severe in Gdf11-null mice than in ActRIIB-null mice, however, leaving it uncertain whether Gdf11 signals via ActRIIB. Here we demonstrate using genetic and biochemical studies that ActRIIB and its subfamily receptor, ActRIIA, cooperatively mediate the Gdf11 signal in patterning the axial vertebrae, and that Gdf11 binds to both ActRIIA and ActRIIB, and induces phosphorylation of Smad2. In addition, we also show that these two receptors can functionally compensate for one another to mediate signaling of another TGF- $\beta$ ligand, nodal, during left-right patterning and the development of anterior head structure.

Received July 9, 2002; revised version accepted September 9, 2002.

Segmentation along the anteroposterior axis is a hallmark of bilaterian development. In vertebrates, somites are formed from the anterior end of the unsegmented presomitic mesoderm. The somites define the segmental boundaries, which provide spatial cues for the develop-

[Keywords: Activin receptor; nodal; Gdf11; vertebrae; left-right asymmetry]

${ }^{5}$ Present address: Department of Life Sciences, Ewha Woman's University, Seoul, Korea 120-750, Korea.

${ }^{6}$ Corresponding author.

E-MAIL ohp@phys.med.ufl.edu; FAX (352) 846-0270.

Article and publication are at http://www.genesdev.org/cgi/doi/10.1101/ gad.1021802. ment of segmental structures such as vertebrae, axial muscles, intercostal blood vessels, and spinal nerve systems. Somites are not morphologically distinguishable along the anterior-posterior axis, and yet each somite acquires a distinct spatial identity as development proceeds. It has been a paradigm that the combinatorial expression of Hox genes in a somite, referred to as the Hox code, determines the anterior-posterior identity of the somite (Kessel and Gruss 1991). However, the molecular mechanisms by which the Hox code is established in mammals remain elusive.

A recent knockout experiment has provided evidence that extracellular signals play an important role in establishing the spatiotemporal pattern of Hox gene expression in somites, thereby specifying vertebral identities. Mice deficient in growth and differentiation factor 11 (Gdf11) show posteriorized expression of several Hox genes and corresponding anterior transformation of the axial skeleton (McPherron et al. 1999). Gdf11 (also known as BMP11) is a secreted protein that belongs to the TGF- $\beta$ superfamily (McPherron et al. 1999). Gdf11 is expressed in the tail bud, limb bud, maxillary and mandibular arches, and dorsal root ganglia during mouse development (Nakashima et al. 1999).

TGF- $\beta$ signals are mediated by heteromeric complexes of type I and type II serine/threonine kinase receptors, which phosphorylate and activate downstream Smad proteins upon ligand stimulation (for review, see Massagué 2000). To date, more than 27 TGF- $\beta$ superfamily ligands have been identified in humans (Venter et al. 2001), whereas only five type II receptors have been discovered in mammals, suggesting that each type II receptor may interact with multiple TGF- $\beta$ ligands. Previous biochemical studies have delineated interactions of these type II receptors with TGF- $\beta$, activin, BMP, or MIS (Mullerian Inhibiting Substance) subfamily proteins (for review, see Piek et al. 1999). Two related type II receptors, ActRIIA and ActRIIB, have been identified as the type II receptors for activins (Mathews and Vale 1991; Attisano et al. 1992). In addition to activins, however, ActRIIA and ActRIIB can biochemically interact with several other TGF- $\beta$ family proteins, including BMP7, Nodal, and Gdf8/Myostatin (Yamashita et al. 1995; Lee and McPherron 2001; Yeo and Whitman 2001). However, in vivo interactions with these signaling partners have yet to be clearly defined. Phenotypic comparison of ligand-deficient mice with receptor-deficient mice, as well as biochemical studies should provide information to delineate TGF- $\beta$ signaling pathways in vivo.

ActRIIA and ActRIIB (designated as IIA and IIB hereafter) receptors share high homology in amino acid sequence, biochemical properties (Mathews and Vale 1991; Attisano et al. 1992), and overlapping expression patterns during development (Feijen et al. 1994). IIB knockout $\left(I I B^{-/-}\right)$mice exhibit multiple patterning defects, including anterior transformation of vertebrae, kidney agenesis, and complex cardiac malformations associated with left-right (LR) asymmetrical defects $(\mathrm{Oh}$ and $\mathrm{Li}$ 1997). IIA knockout $\left(I I A^{-/-}\right)$mice are mostly normal, but some show mandibular hypoplasia, reduced fertility, and gastrulation defects (Matzuk et al. 1995; Song et al. 1999). ActRIIA and ActRIIB genes can also functionally compensate one another in regulating gastrulation, foregut patterning, and tooth development (Song et al. 1999; 
Kim et al. 2000; Ferguson et al. 2001). The vertebral transformation in $I I B^{-/-}$mice is reminiscent of, but less severe than, that of $G d f 11^{-/-}$mice. This suggests that ActRIIB might be a receptor for Gdf11, and if it is, there must be another receptor to mediate Gdf11 during the specification of vertebral pattern /Gad and Tam 1999|. In this study, we provide genetic and biochemical evidence that ActRIIA and ActRIIB cooperatively mediate the Gdf11 signal for the specification of the axial vertebrae.

\section{Results and Discussion}

To investigate the compensatory role of IIA in $I I B^{-/-}$ mice, we bred $I I A^{+/-} I I B^{+/-}$mice with either $I I B^{-/-}$or $I I B^{+/-}$mice on a 129SvJae/C57BL6 hybrid background and compared the phenotypes of $I I A^{+/-} I I B^{-/-}$embryos with those of $I I B^{-/-}$littermates at birth or from embryonic day 10.5 to 18.5 (E10.5-E18.5). $I I A^{+/-} I I B^{-/-}$mice showed a higher frequency of mortality at $\sim \mathrm{E} 14.5$ as compared with $I I B^{-/-}$mice. However, the majority of $I I A^{+7-} I I B^{-1-}$ mice developed to term and exhibited multiple defects in body patterning and organogenesis, which included anterior transformation of the vertebral skeleton, cleft palate, kidney agenesis, right pulmonary isomerism accompanied by cardiac malformation, and truncation of anterior head structures (Table 1). Overall, the $I I A^{+/-} I I B^{-/-}$mice showed a dramatic increase in both the severity and penetrance of the mutant phenotypes as compared with $I I B^{-/-}$mice, suggesting that IIA can partially compensate for the loss of IIB during the morphogenesis of multiple organs in mice.

The mouse vertebral column consists of seven cervical (C), 13 thoracic (T), six lumbar (L), three or four sacral, and the caudal vertebrae, displaying the C7 T13 L6 pattern (Fig. 1A). The thoracic vertebrae are characterized by their attachment to ribs, and the first seven ribs are attached to the sternum, referred to as vertebrosternal (VS) ribs (Fig. 1D). As shown previously, vertebrae of the $I I B^{-/-}$mice had a homeotic transformation, displaying the C7 T16 L6 pattern with nine pairs of VS ribs (Fig. $1 \mathrm{~B}, \mathrm{E}$; $\mathrm{Oh}$ and $\mathrm{Li} 1997)$. We found that $I I A^{+/-} I I B^{-/-}$mice displayed additional vertebral transformations, resulting in the C7 T17 L7 pattern with 10 pairs of VS ribs (Table 1; Fig. $1 \mathrm{C}, \mathrm{F})$. In $\sim 80 \%$ of $I I A^{+/-} I I B^{-/-}$mice, the first rib from $\mathrm{T} 1$ was fused ventrally to the second rib from T2, and the first VS rib was disconnected to T1 (Table 1; Fig. $1 F)$. The anterior transformation of the axial skeleton was also found in cervical vertebrae of $I I A^{+/-} I I B^{-/-}$mice. The seventh cervical vertebra (C7) of $I I A^{+/-} I I B^{-/-}$mice acquired the morphology of C6: The tuberculi anterior, a morphological marker of $\mathrm{C} 6$, and the transverse foramen, a structure found in the C4-C6 vertebrae, were found in C7 of $I I A^{+/-} I I B^{-/-}$mice (Table 1; Fig. 1I,L).

We also observed several forms of herniation in $I I A^{+/-}$ $I I B^{-/-}$mice, which might be caused by the vertebral anterior transformation. In some $I I A^{+/-} I I B^{-/-}$mice, the stomach was mislocated in the thoracic cavity above the diaphragm (diaphragmatic herniation; Fig. 2B), or the abdominal organs were protruded outside of the abdomen (body wall herniation; Fig. 2D). We speculate that the additional vertebral transformation in $I I A^{+/-} I I B^{-/-}$mice may cause such herniations by further lowering the diaphragm, which increases the extent of the thoracic cavity while reducing the abdominal cavity. In addition, we also found that about a half of the $I I A^{+/-} I I B^{-/-}$mice had
Table 1. Comparison of axial skeletal and organogenesis defects between $I I B^{-/-}$and $I I A^{+/-} I I B^{-/-}$mice

\begin{tabular}{|c|c|c|}
\hline & $I I B^{-/-}$ & $I I A^{+/-} I I B^{-/-}$ \\
\hline \multicolumn{3}{|l|}{ Vertebral patterning } \\
\hline C7 T16 L5 & $5 / 27$ & $0 / 27$ \\
\hline C7 T16 L6 & $22 / 27$ & $0 / 27$ \\
\hline C7 T17 L6 & $0 / 27$ & $4 / 27$ \\
\hline C7 T17 L7 & $0 / 27$ & $20 / 27$ \\
\hline C7 T18 L6 & $0 / 27$ & $3 / 27$ \\
\hline \multicolumn{3}{|l|}{ Vertebrosternal ribs } \\
\hline 8 & $1 / 27$ & $0 / 27$ \\
\hline 9 & $26 / 27$ & $1 / 27$ \\
\hline 10 & $0 / 27$ & $26 / 27$ \\
\hline Fusion of $\mathrm{T} 1 / \mathrm{T} 2$ ribs & $4 / 27$ & $22 / 27$ \\
\hline \multicolumn{3}{|l|}{ Tuberculi anterior on } \\
\hline C6 only & $23 / 27$ & $5 / 27$ \\
\hline $\mathrm{C} 6$ and $\mathrm{C} 7$ & $4 / 27$ & $14 / 27$ \\
\hline none & $0 / 27$ & $6 / 27$ \\
\hline C7 only & $0 / 27$ & $2 / 27$ \\
\hline Cleft palate & $1 / 80(1 \%)$ & $25 / 55(51 \%)$ \\
\hline Tail defects & $0 / 80(0 \%)$ & $31 / 55(56 \%)$ \\
\hline (D) herniation & $0 / 80(0 \%)$ & $7 / 55(13 \%)$ \\
\hline (BW) herniation & $0 / 80(0 \%)$ & $5 / 55(9 \%)$ \\
\hline Kidney defects & $19 / 76(25 \%)$ & $48 / 49(98 \%)$ \\
\hline no kidney & $6 / 19$ & $39 / 48$ \\
\hline unilateral agenesis & $8 / 19$ & $4 / 48$ \\
\hline hypoplastic & $5 / 19$ & $5 / 48$ \\
\hline RPI/TGA & $38 / 80(48 \%)$ & $55 / 55(100 \%)$ \\
\hline atrial isomerism & $25 / 38(66 \%)$ & $55 / 55(100 \%)$ \\
\hline dextrocardia & $5 / 38(13 \%)$ & $24 / 55(44 \%)$ \\
\hline bilateral IVC & $2 / 38(5 \%)$ & $15 / 34(44 \%)$ \\
\hline hypo. spleen & $1 / 38(3 \%)$ & $23 / 45(51 \%)$ \\
\hline Anterior head defects & $0 / 80(0 \%)$ & $5 / 55(9 \%)$ \\
\hline Open eye & $0 / 80(0 \%)$ & $6 / 55(11 \%)$ \\
\hline
\end{tabular}

Abbreviations: $\mathrm{D}$, diaphragmatic; $\mathrm{BW}$, body wall; RPI, right pulmonary isomerism; TGA, transposition of great arteries; IVC, inferior vena cava.

short or curly tails or no tails (Fig. 2F-H), indicating an important role for IIA and IIB in development of the caudal vertebrae. These findings indicate that although the IIA gene itself is not required for the normal AP patterning of the axial skeleton during development (Matzuk et al. 1995), it does have a compensatory role for the IIB receptor in mediating signals for vertebral specification and caudal development.

The phenotype of vertebral transformation in $I I B^{-/-}$ mice is remarkably similar to, although less severe than, that of $G d f 11^{-/-}$mice, which exhibit the C7 T18 L8 pattern with 10 VS ribs, C7 transformation, and tail defects (McPherron et al. 1999). The differences in severity suggest that $I I B^{-/-}$only partially abrogates the Gdf11 signal and there must exist another receptor that mediates the Gdf11 signal in vertebral patterning. The vertebral defects of $I I A^{+/-} I I B^{-/-}$mice are almost identical to those of $G d f 11^{-/-}$mice, suggesting that IIA is the other receptor mediating the Gdf11 signal. To exclude the possibility that Gdf11 might be a downstream effector rather than the ligand of activin type II receptors, we examined 


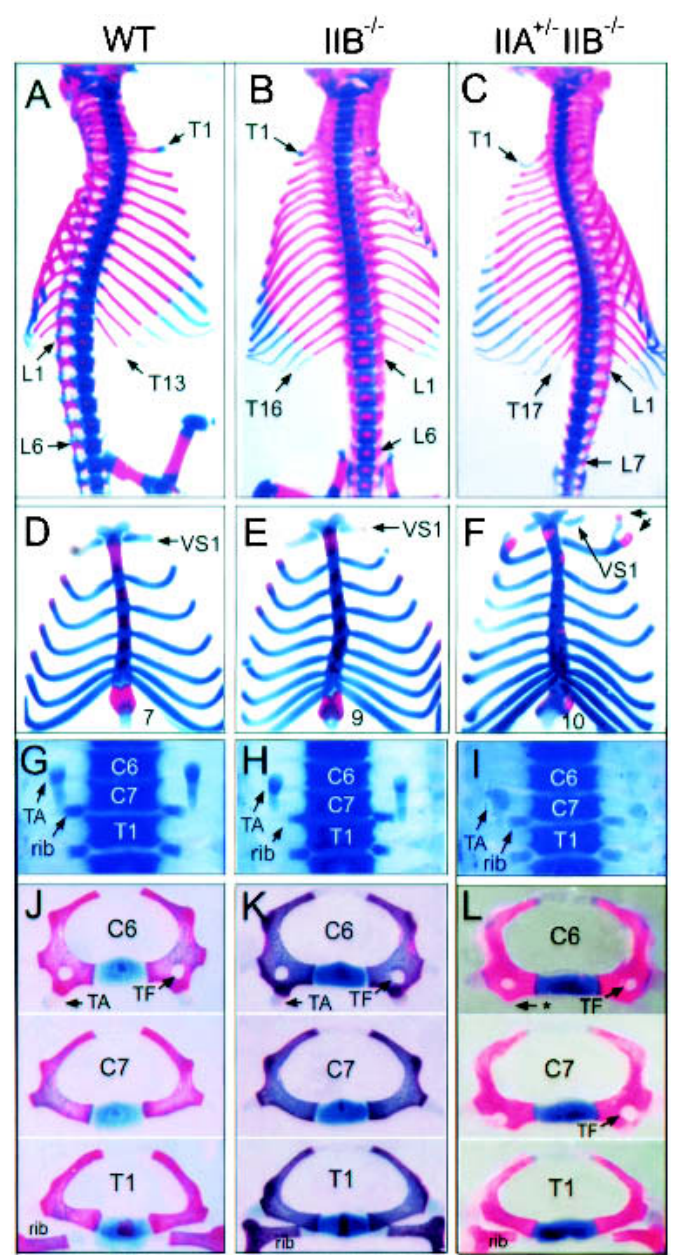

Figure 1. Axial vertebral patterning in activin receptor mutant mice. Representative vertebral patterning in wild-type $(A, D, G, J)$, $I I B^{-/-}(B, E, H, K)$, and $I I A^{+/-} I I B^{-/-}(C, F, I, L)$ mice. $(A-C)$ Ventral view of vertebral skeletons. Wild-type skeleton consists of 13 thoracic $(\mathrm{T})$ and 6 lumbar (L) vertebrae. The skeleton pattern is altered to T16L6 in $I I B^{-/-}$and T17L7 in $I I A^{+/-} I I B^{-/-}$mice. $(D-F)$ Increased number of vertebrosternal (VS) ribs in $I I B^{-/-}$and $I I A^{+/-} I I B^{-/-}$mice. Arrows in $F$ indicate $\mathrm{T} 1$ and $\mathrm{T} 2$ ribs fused ventrally to VS2 rib. $(G-I)$ Cervical and thoracic vertebrae showing transformation of $\mathrm{C} 7$ vertebra in $I I A^{+/-}$ $I I B^{-/-}$mice. Tuberculi anterior (TA) is present at $\mathrm{C} 6$ in wild-type and $I I B^{-/-}$mice, whereas it is present at $\mathrm{C} 7$ in $I I A^{+/-} I I B^{-/-}$mice. Attached ribs indicate thoracic vertebrae. $(J-L)$ Morphology of C6-T1 vertebrae showing $\mathrm{C} 7$ to $\mathrm{C} 6$ transformation in $I I A^{+/-} \mathrm{IIB}^{-/-}$mice. Note that the tuberculi anterior is missing in $\mathrm{C} 6$ (asterisk) and the transverse foramen (TF) is present in $\mathrm{C} 7$ of $I I A^{+/-} I I B^{-1-}$ mice.

Gdf11 expression in $I I B^{-/-}$and $I I A^{+/-} I I B^{-/-}$embryos at E9.5-E11.5, using whole-mount in situ hybridization. We found no difference in the expression pattern and intensity of $G d f 11$ transcripts between the $I I A^{+/-} I I B^{-/-}$ embryos and the wild-type littermates (data not shown).

In addition to the skeletal defects, $G d f 11^{-/-}$mice also exhibit defects in kidney and palate formation (McPherron et al. 1999). Consistent with this, $I I A^{+/-} I I B^{-/-}$mice also displayed a significantly increased frequency and severity of defects in kidney and palate formation as compared with $I I B^{-/-}$mice. Cleft palate was rarely observed in $I I B^{-/-}$mice $(1 / 80)$, but was common among $I I A^{+/-}$ $I I B^{-/-}$mice $(50 \%, 27 / 53$; Table 1; Fig. 2J). Similarly, the incidence of kidney defects was increased from $26 \%$ in $I I B^{-/-}$to $98 \%$ in $I I A^{+/-} I I B^{-/-}$mice (Table 1; Fig. $2 \mathrm{~L}$ ), and a considerably higher percentage $(80 \%)$ of $I I A^{+/-} I I B^{-/-}$ mice showed bilateral kidney agenesis (Table 1).

We also observed developmental defects such as right isomerism and anterior head defects that were not seen in $G d f 11^{-/-}$mice. The right isomerism is characterized by systematic heterotaxia, including bilateral mirror image of right lung patterns, randomization of heart positions, bilateral right atria, bilateral inferior vena cava (IVC), absence or hypoplasia of spleen, and randomization of asymmetric patterns of abdominal organs (Oh and Li 2002). About $48 \%$ (38/80) of $I I B^{-/-}$mice on the $129 \mathrm{~Sv} /$ C57BL6 hybrid background displayed right pulmonary isomerism (RPI) characterized by bilateral tetralobed lungs and associated systematic alterations in heart and other organs (Table. 1). In their $I I A^{+/-} I I B^{-/-}$littermates, however, the frequency of RPI was $100 \%$ (55/55), and penetrance of other characteristic defects, including atrial isomerism, dextrocardia, hypoplasia of spleen, and persistent left hepatic vein (i.e., bilateral inferior vena cava), was significantly increased (Table 1).

The head defects observed in $I I A^{+/-} I I B^{-/-}$pups include the truncation of anterior head structures and fusion of the eyes (cyclopia) to variable degrees (Fig. 2F-H). Whereas the axial skeletal defect, kidney agenesis, and cleft palate appear to be caused by impaired Gdf11 signaling, the anterior head defects are probably caused by

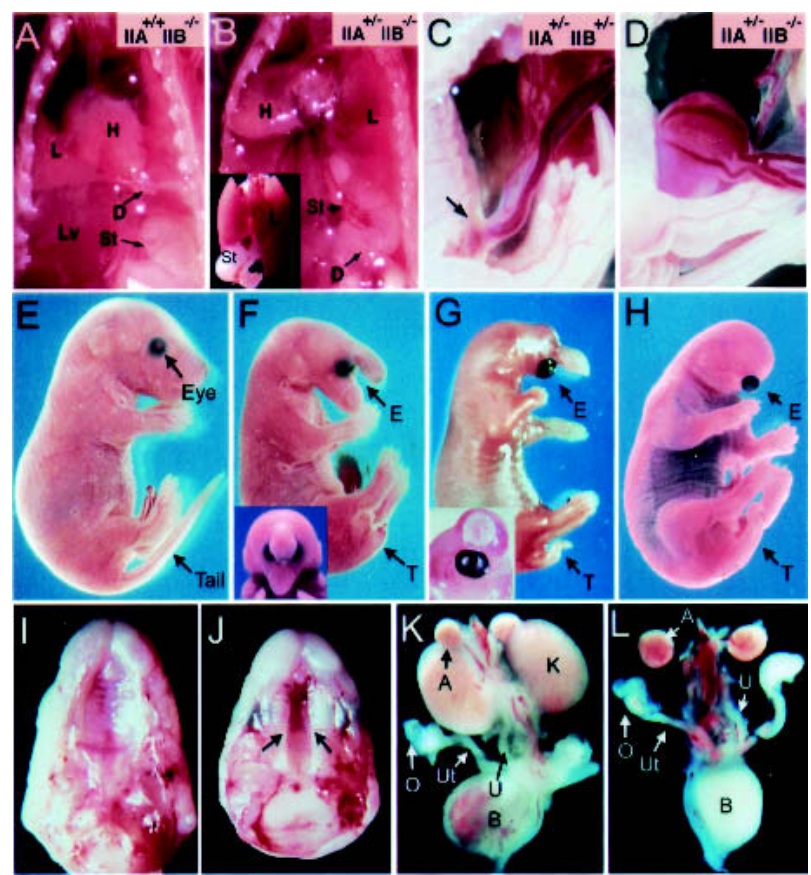

Figure 2. Multiple developmental defects in $I I A^{+/-} \mathrm{IIB}^{-/-}$newborns. $(A, B)$ Diaphragmatic herniation in $I I A^{+/-} I I B^{-/-}$newborn. The stomach and spleen are located in the abdominal cavity, below the diaphragm in wild-type or $I I B^{-/-}$mice $(A)$. In some $I I A^{+/-} I I B^{-/-}$mice, the stomach and hypoplastic spleen were mislocated above the diaphragm $(B)$. (Inset) The stomach and spleen located behind the lung. $\mathrm{D}$, diaphragm; L, lung; Lv, liver; St, stomach. $(C, D)$ Body wall herniation in E18.5 $I I A^{+/-} I I B^{-1-}$ fetus $(D)$. Arrows indicate region of the umbilical ring. $(E-H)$ Lateral view of a wild-type $(E)$ and three $I I A^{+/-}$ $I I B^{-/-}(F-H)$ newborn pups, displaying variable defects in anterior head, cyclopia, and tail formation. (F,G insets) The frontal view of the corresponding mutants. $(I-L)$ Cleft palate $(J)$ and bilateral kidney agenesis $(L)$ in $I I A^{+/-} I I B^{-/-}$mutant mice. The arrows in $J$ indicate cleft palate. A, adrenal gland; B, bladder; $\mathrm{K}$, kidney; O, ovary; $\mathrm{Ut}$, uterine; U, urethra. 
Oh et al.

disrupting the nodal signaling pathway. Our previous studies showed that nodal and IIA double mutants (nodal ${ }^{+/-} I I A^{-/-}$) and nodal and Smad double mutants $\left(\right.$ nodal $\left.^{+/}-\mathrm{Smad}^{+/-}\right)$displayed anterior head truncation and cyclopia (Nomura and Li 1998; Song et al. 1999). Similarly, zebrafish mutants defective in nodal signaling (cyc, sqt, or oep) also display cyclopia, indicating that nodal is necessary for forebrain and midline development (for review, see Schier and Shen 2000; Schier and Talbot 2001; Whitman 2001).

Using a Xenopus embryo assay system, it has been shown that the nodal signal can be transduced by IIB in association with the coreceptor Cripto and the type I receptor ALK4 to activate Smad2 phosphorylation (Yeo and Whitman 2001). To obtain biochemical evidence that the Gdf11 signal was indeed transduced by IIA and IIB, we used this Xenopus assay system. We first examined whether Gdf11 could induce phosphorylation of Smad2. As shown in Figure 3A, Gdf11 stimulated phosphorylation of Smad2 and suppressed endogenous Smad1 phosphorylation, when ectopically expressed in Xenopus ectodermal explants. This result indicates that Gdf11 likely functions via TGF- $\beta$ or activin type II receptors, but not via BMP type II receptors. To determine the binding specificity of Gdf11 to different receptors, Gdf11 and Gdf10 were tagged with a Flag epitope at the $\mathrm{N}$ terminus of the mature region. Gdf10 was used as a negative control as it likely acts through the BMP receptors (Cunningham et al. 1995). To examine whether Flag-tagged Gdf11 is properly processed and functions as an active ligand, we analyzed its ability to activate Smad2. Both Gdf11 and Flag-Gdf11, but not Gdf10, were able to induced Smad2 phosphorylation in Xenopus ectodermal explants (Fig. 3B).

We then examined Gdf11 binding to activin receptor complexes by coimmunoprecipitation of Flag-tagged Gdf11 with HA (or Myc) -tagged, kinase-defective IIA/ ALK4 or IIB/ALK4. Kinase-defective receptors were used to minimize receptor down-regulation and/or complex dissociation. When ALK4 was coexpressed with IIA or IIB, Gdf11, but not Gdf10, was coprecipitated with ALK4, indicating that the activin receptor complexes specifically interact with Gdf11 (Fig. 3C). It is interesting to note that IIA was only weakly coprecipitated with ALK4 in the absence of Gdf11, whereas IIB was coprecipitated with ALK4 regardless of Gdf11, suggesting a different binding property of IIA and IIB to ALK4 (Fig. 3C). We further showed that Gdf11 was coprecipitated with IIA or IIB regardless of ALK4 expression (Fig. 3D). In a similar experimental scheme, Gdf11 did not coprecipitate with the TGF- $\beta$ type II receptor (data not shown), indicating that Gdf11 specifically binds to activin IIA and IIB receptors. Interestingly, the Gdf11 cross-linking signal was consistently more intense when Gdf11 was coexpressed with IIB than with IIA, indicating a higher binding property of IIB than IIA for Gdf11 (Fig. 3D). Only a very weak Gdf11 band was detected when Gdf11 was coexpressed with ALK4 alone. The weak band might be due to the presence of the endogenous activin type II receptors, and indicates that Gdf11 does not bind strongly to ALK4 in the absence of the activin Type II receptor. We did not observe a synergy between IIA and IIB in their binding to Gdf11: Gdf11 binding was increased by coexpression of IIA and IIB as compared with IIA alone, but it was decreased as compared with IIB alone (Fig. 3D).

Genetic studies have shown that the nodal signaling pathway plays a crucial role in at least four developmental processes: mesoderm formation (Zhou et al. 1993; Conlon et al. 1994), primitive streak elongation (Song et al. 1999; Lowe et al. 2001; Yamamoto et al. 2001), anterior head formation (Varlet et al. 1997; Nomura and Li 1998; Song et al. 1999; Schier and Talbot 2001), and leftright patterning (Yan et al. 1999; Lowe et al. 2001; Oh and Li 2002). Genetic crosses of IIA and IIB knockout mice in this and previous studies have demonstrated a strong phenocopy of all four nodal-related phenotypes in the IIA/IIB compound mutant mice /Oh and Li 1997;
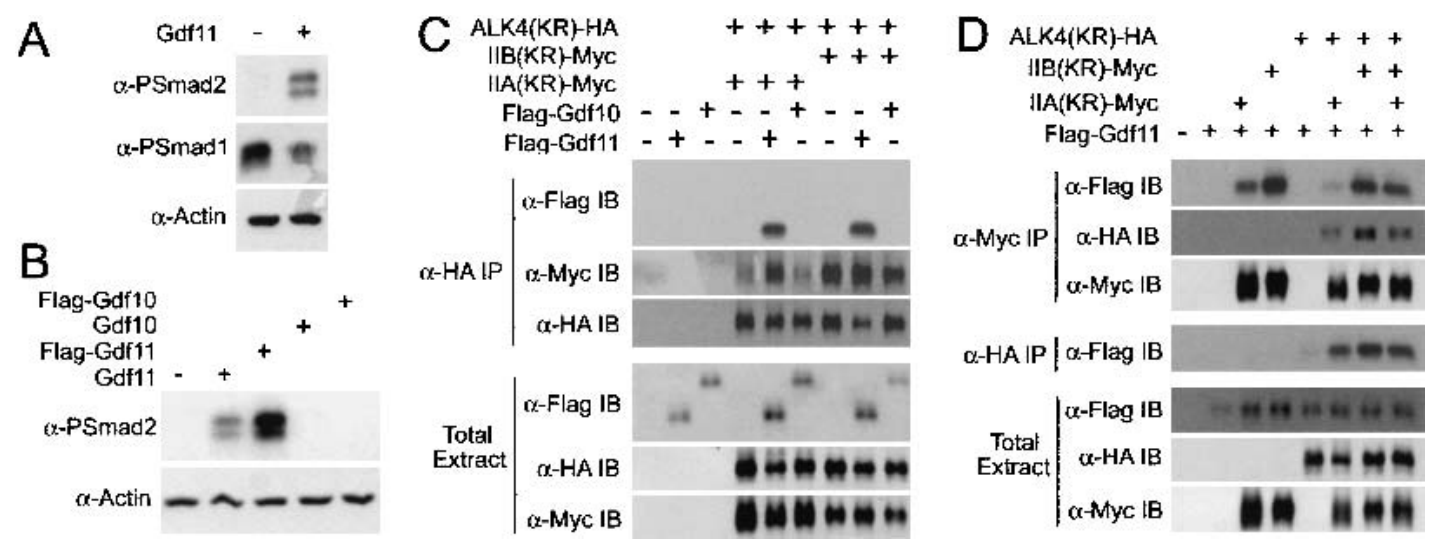

Figure 3. Gdf11 binds to IIA and IIB and phosphorylates Smad2. (A) Gdf11 can induce Smad2 phosphorylation. Activated Smad2 and suppressed Smad1 phosphorylation were detected by anti-phospho-Smad1 ( $\alpha$-PSmad1) or anti-phospho-Smad2 antibodies $(\alpha$-PSmad2) in stage 10 ectodermal explants of Xenopus embryos. Cytoskeletal actin was used as a loading control ( $\alpha$-Actin). (B) Both Gdf11 and Flag-Gdf11, but neither Gdf10 nor Flag-Gdf10, induced Smad2 phosphorylation, indicating that Flag-Gdf11 is functionally active. (C) Coimmunoprecipitation analyses showing that Gdf11, but not Gdf10, interacts with the activin receptor complexes (top panel). Note that IIA coprecipitated with ALK4 effectively only in the presence of Gdf11, whereas IIB did so regardless of the ligand (second panel). Comparable levels of protein expression in total extracts are shown. $(D)$ Coimmunoprecipitation analyses showing that Gdf11 binds to IIA and IIB to different degrees. Both IIA and IIB were coprecipitated with Gdf11 in the absence or presence of ALK4 (top panel), whereas ALK4 was coprecipitated with Gdf11 only in the presence of type II receptors (fourth panel). Note that the amount of Gdf11 coprecipitated with IIA was smaller than that with IIB (top panel). For all experiments, $2 \mathrm{ng}$ of $I I A(K R)-M y c$ or $I I B(K R)-M y c$ mRNA was injected, except for the last lane in which $1 \mathrm{ng}$ each of $I I A$ and $I I B$ mRNA was injected instead. 
Song et al. 1999|, suggesting that the nodal signal is mediated by activin IIA and IIB receptors. In addition to these nodal-related phenotypes, the IIA/IIB compound mutant mice exhibited the Gdf11-related phenotypes, including vertebral homeotic transformation, tail defects, cleft palate, and kidney defects (McPherron et al. 1999).

Although IIA and IIB have overlapping functions, their roles in many development processes are not equal in that one receptor has a primary role whereas the other has a supplementary role. For Gdf11 signaling, IIB clearly functions as the primary receptor and IIA as the supplementary receptor, because IIA by itself is neither required for the vertebral patterning, nor for the morphogenesis of kidney, palate, and tail (Matzuk et al. 1995; Song et al. 1999). Our observations indicate that the relatively mild phenotype or low penetrance of defects in vertebral patterning, kidney morphogenesis, and palate and tail development in $I I B^{-/-}$mice is caused by partial compensation by IIA. However, the compensatory effect of IIA is markedly reduced when one IIA allele is mutated as in $I I A^{+/-} I I B^{-/-}$mice. Conversely, IIA plays the primary role and IIB the supplementary role in mediating the nodal signal for primitive streak elongation. IIB is dispensable for primitive streak elongation as all $I I B^{-/-}$mice develop to term $/ \mathrm{Oh}$ and $\mathrm{Li}$ 1997). In the absence of IIA, IIB can partially compensate for the loss of IIA in primitive streak elongation as most $I I A^{-/-}$mice develop to term. However, a single copy of the wild-type $I I B$ allele is not sufficient to compensate for the loss of IIA in $I I A^{-/-} I I B^{+/-}$embryos, most of which are defective in primitive streak elongation (Song et al. 1999).

How are the primary and supplementary roles of IIA and IIB assigned during development? One possibility is that the spatial and temporal expression pattern of these receptors with respect to a given ligand determines which receptor has the primary role. In this case the primary receptor may be sufficiently abundant in critical tissues to allow optimal signaling, whereas the supplementary receptor is expressed at lower levels that are not sufficient to allow optimal signaling for a specific developmental process in the absence of the primary receptor. For instance, strong expression of IIB, but weak expression of IIA in the metanephros (Feijen et al. 1994) may explain the primary role of IIB in kidney development.

The second possibility is that the IIA and IIB receptors differ in their relative affinities for a given ligand or a type I receptor. Biochemical analysis of ligands and receptors expressed in the Xenopus embryos revealed that Gdf11 binds more efficiently to the IIB/ALK4 than to the IIA/ALK4 complexes. We also observed that IIA binding to ALK4 is Gdf11-dependent, whereas IIB is not. The identification of coreceptors that can modulate signaling by TGF- $\beta$ superfamily ligands during embryonic development (Reissmann et al. 2001; Yeo and Whitman 2001; Yan et al. 2002) provides additional complexity as to how ligand-receptor specificity is determined.

What other developmental processes might be regulated by IIA and IIB signaling pathways, and what other TGF- $\beta$ family ligands might signal through IIA and IIB receptors? Because $I I A^{-/-} I I B^{+/-}$mice are embryonic lethal, conditional inactivation of the IIA gene using the Cre-loxP system will be necessary to further investigate the functions of IIA and IIB during late development and in adult mice. Phenotype comparison between ligand and receptor mutants and biochemical studies of signaling of a given ligand through IIA and IIB receptors and coreceptors such as Cripto and Criptic will shed further light on ligand-receptor specificity within the TGF- $\beta$ superfamily during mouse development.

\section{Materials and methods}

Mouse strains and crosses

We have previously reported detailed methods for the generation of IIA and IIB-KO mice (Oh and Li 1997; Song et al. 1999). Anatomical criteria for phenotypes related to the laterality defect in newborn pups were as described (Oh and Li 2002). Genotypes of individual pups were performed by PCR analysis as described (Song et al. 1999).

Phospho-Smad2 analysis

All synthetic mRNAs were transcribed from cDNAs in the pCS2+ vector or its derivatives, using the SP6 mMessage mMachine Kit (Ambion). Plasmids pCS-Flag-Gdf11 and pCS-Flag-Gdf10 contain the proregion of chick Dorsalin (codons 1 to Ala 322; Constam and Robertson 1999), three repeats of Flag epitope, and the mature region of human Gdf11 (from Asn299) or mouse Gdf10 (from Lys338). Synthetic mRNA (1 ng of each RNA/embryo) was injected into each blastomere in the animal hemisphere of 2- to 4-cell Xenopus embryos. Ectodermal explants were isolated when uninjected siblings were at stages 8-9 and harvested at stage 10. Western blot analysis of Smad2 phosphorylation was performed as described (Yeo and Whitman 2001).

\section{Coimmunoprecipitation analysis}

Plasmids pCS-IIA(KR)-Myc and pCS-IIB(KR)-Myc encode kinase-defective mutants of mouse ActRIIA (Lys 219 to Arg) or mouse ActRIIB (Lys 217 to Arg), respectively, followed by six repeats of Myc epitope. pCSALK4(KR)-HA encodes a kinase-defective mutant of human ALK4 (Lys 234 to Arg) with six repeats of HA epitope. Synthetic mRNAs encoding IIA(KR)-Myc, IIB(KR)-Myc, ALK4(KR)-HA, or Flag-Gdf11 (2 ng of each RNA/embryo, unless otherwise specified) were injected into each blastomere at the animal hemisphere of 2- to 4-cell Xenopus embryos. Embryos were bisected along the animal-vegetal axis when uninjected sibling embryos reached stage 10 . To cross-link extracellular proteins, bisected embryos were incubated with $20 \mathrm{mM}$ DTSSP (Pierce) in PBS at $4^{\circ} \mathrm{C}$ for $2 \mathrm{~h}$. Embryos were then harvested and used for immunoprecipitation. Total extracts and immunoprecipitated proteins were treated with PNGase F (New England BioLabs) to remove N-linked glycans from proteins and then with $5 \mathrm{mM}$ DTT to cleave DTSSP. Immunoprecipitation and Western blot analysis were performed as described (Yeo and Whitman 2001).

\section{Acknowledgments}

We thank S-J. Lee for the Gdf11 in situ probe and Gdf11 and Gdf10 full-length cDNAs, and P. Sayeski and S. Park for critical comments on the manuscript. This work was supported by NIH grants HL64024 to S.P.O., HD24926 to M.W., and HD35286 to E.L.

The publication costs of this article were defrayed in part by payment of page charges. This article must therefore be hereby marked "advertisement" in accordance with 18 USC section 1734 solely to indicate this fact.

\section{References}

Attisano, L., Wrana, J.L., Cheifetz, S., and Massague, J. 1992. Novel activin receptors: Distinct genes and alternative mRNA splicing generate a repertoire of serine/threonine kinase receptors. Cell 68: 97108.

Conlon, F.L., Lyons, K.M., Takaesu, N., Barth, K.S., Kispert, A., Herrmann, B., and Robertson, E.J. 1994. A primary requirement for nodal in the formation and maintenance of the primitive streak in the mouse. Development 120: 1919-1928.

Constam, D.B. and Robertson, E.J. 1999. Regulation of bone morphogenetic protein activity by pro domains and proprotein convertases. $J$. Cell Biol. 144: 139-149. 
Oh et al.

Cunningham, N.S., Jenkins, N.A., Gilbert, D.J., Copeland, N.G., Reddi, A.H., and Lee, S.J. 1995. Growth/differentiation factor-10: A new member of the transforming growth factor- $\beta$ superfamily related to bone morphogenetic protein-3. Growth Factors 12: 99-109.

Feijen, A., Goumans, M.J., and van den Eijnden-van Raaij A.J. 1994. Expression of activin subunits, activin receptors and follistatin in postimplantation mouse embryos suggests specific developmental functions for different activins. Development 120: 3621-3637.

Ferguson, C.A., Tucker, A.S., Heikinheimo, K., Nomura, M., Oh, S.P., Li, E., and Sharpe, P.T. 2001. The role of effectors of the activin signalling pathway, activin receptors IIA and IIB, and Smad2, in patterning of tooth development. Development 128: 4605-4613.

Gad, J.M. and Tam, P.P. 1999. Axis development: The mouse becomes a dachshund. Curr. Biol. 9: R783-R786.

Kessel, M. and Gruss, P. 1991. Homeotic transformations of murine vertebrae and concomitant alteration of Hox codes induced by retinoic acid. Cell 67: 89-104.

Kim, S.K., Hebrok, M., Li, E., Oh, S.P., Schrewe, H., Harmon, E.B., Lee, J.S., and Melton, D.A. 2000. Activin receptor patterning of foregut organogenesis. Genes \& Dev. 14: 1866-1871.

Lee, S.J. and McPherron, A.C. 2000. Regulation of myostatin activity and muscle growth. Proc. Natl. Acad. Sci. 98: 9306-9311.

Lowe, L.A., Yamada, S., and Kuehn, M.R. 2001. Genetic dissection of nodal function in patterning the mouse embryo. Development 128: 1831-1843.

Massagué, J. 2000. How cells read TGF- $\beta$ signals. Nat. Rev. Mol. Cell Biol. 1: 169-178.

Mathews, L.S. and Vale, W.W. 1991. Expression cloning of an activin receptor, a predicted transmembrane serine kinase. Cell 65: 973-982.

Matzuk, M.M., Kumar, T.R., and Bradley, A. 1995. Different phenotypes for mice deficient in either activins or activin receptor type II. Nature 374: $356-360$.

McPherron, A.C., Lawler, A.M., and Lee, S.J. 1999. Regulation of anterior/posterior patterning of the axial skeleton by growth/differentiation factor 11. Nat. Genet. 22: 260-264.

Nakashima, M., Toyono, T., Akamine, A., and Joyner, A. 1999. Expression of growth/differentiation factor 11, a new member of the BMP/ TGF $\beta$ superfamily during mouse embryogenesis. Mech. Dev. 80: 185189.

Nomura, M. and Li, E. 1998. Smad2 role in mesoderm formation, leftright patterning and craniofacial development. Nature 393: 786-790.

Oh, S.P. and Li, E. 1997. The signaling pathway mediated by the type IIB activin receptor controls axial patterning and lateral asymmetry in the mouse. Genes \& Dev. 11: 1812-1826.

- 2002. Gene-dosage-sensitive genetic interactions between inversus viscerum (iv), nodal, and activin type IIB receptor (ActRIIB) genes in asymmetrical patterning of the visceral organs along the left-right axis. Dev. Dynamics 224: 279-290.

Piek, E., Heldin, C.H., and ten Dijke, P. 1999. Specificity, diversity, and regulation in TGF- $\beta$ superfamily signaling. FASEB J. 13: 2105-2124.

Reissmann, E., Jornvall, H., Blokzijl, A., Andersonn, O., Chang, C., Minchiotti, G., Persico, M.G., Ibanez, C.F., and Brivanlou, A.H. 2001. The Orphan receptor ALK7 and the Activin receptor ALK4 mediate signaling by Nodal proteins during vertebrate development. Genes \& Dev. 15: 2010-2022

Schier, A.F. and Shen, M.M. 2000. Nodal signalling in vertebrate development. Nature 403: 385-389.

Schier, A.F. and Talbot, W.S. 2001. Nodal signaling and the zebrafish organizer. Intl. J. Dev. Biol. 45: 289-297.

Song, J., Oh, S.P., Schrewe, H., Nomura, M., Lei, H., Okano, M., Gridley, T., and Li, E. 1999. The type II activin receptors are essential for egg cylinder growth, gastrulation, and rostral head development in mice. Dev. Biol. 213: 157-169.

Varlet, I., Collignon, J., and Robertson, E.J. 1997. Nodal expression in the primitive endoderm is required for specification of the anterior axis during mouse gastrulation. Development 124: 1033-1044.

Venter, J.C., Adams, M.D., Myers, E.W., Li, P.W., Mural, R.J., Sutton, G.G., Smith, H.O., Yandell, M., Evans, C.A., Holt, R.A., et al. 2001 The sequence of the human genome. Science 291: 1304-1351.

Whitman, M. 2001. Nodal signaling in early vertebrate embryos: Themes and variations. Dev. Cell 1: 605-617.

Yamamoto, M., Meno, C., Sakai, Y., Shiratori, H., Mochida, K., Ikawa, Y., Saijoh, Y., and Hamada, H. 2001. The transcription factor FoxH1
(FAST) mediates Nodal signaling during anterior-posterior patterning and node formation in the mouse. Genes \& Dev. 15: 1242-1256.

Yamashita, H., Ten Dijke, P., Huylebroeck, D., Sampath, T.K., Andries, M., Smith, J.C., Heldin, C.H., and Miyazono, K. 1995. Osteogenic protein-1 binds to activin type II receptors and induces certain activin-like effects. J. Cell Biol. 130: 217-226.

Yan, Y.T., Gritsman, K., Ding, J., Burdine, R.D., Corrales, J.D., Price, S.M., Talbot, W.S., Schier, A.F., and Shen, M.M. 1999. Conserved requirement for EGF-CFC genes in vertebrate left-right axis formation. Genes \& Dev. 13: 2527-2537.

Yan, Y.T., Liu, J.-J., Luo, Y., Chaosu, E., Haltiwanger, R.S., Abate-Shen, C., and Shen, M.M. 2002. Dual roles of Cripto as a ligand and coreceptor in the nodal signaling pathway. Mol. Cell. Biol. 22: 44394449.

Yeo, C. and Whitman, M. 2001. Nodal signals to Smads through Criptodependent and Cripto-independent mechanisms. Mol. Cell 7: 949957.

Zhou, X., Sasaki, H., Lowe, L., Hogan, B.L., and Kuehn, M.R. 1993. Nodal is a novel TGF- $\beta$-like gene expressed in the mouse node during gastrulation. Nature 361: 543-547. 


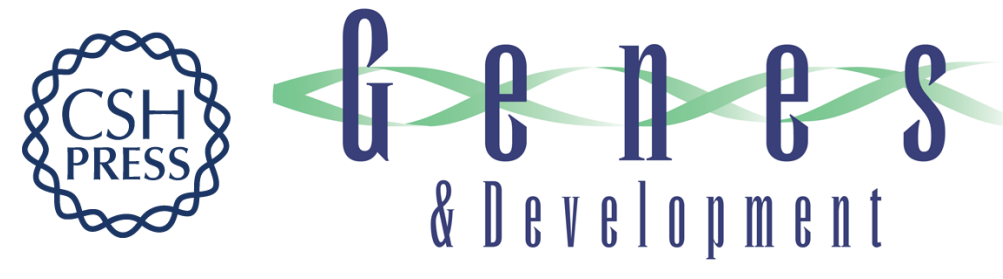

\section{Activin type IIA and IIB receptors mediate Gdf11 signaling in axial vertebral patterning}

S. Paul Oh, Chang-Yeol Yeo, Youngjae Lee, et al.

Genes Dev. 2002, 16:

Access the most recent version at doi:10.1101/gad.1021802

References This article cites 33 articles, 15 of which can be accessed free at: http://genesdev.cshlp.org/content/16/21/2749.full.html\#ref-list-1

License

Email Alerting Receive free email alerts when new articles cite this article - sign up in the box at the top Service right corner of the article or click here.

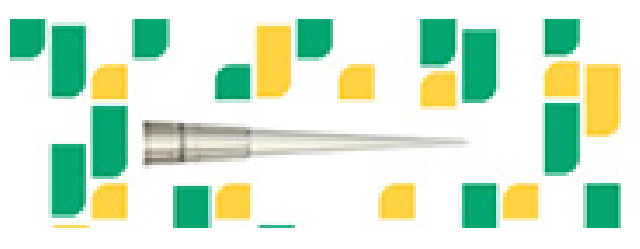

Focused on your science. 